\title{
Development of quality standards in inflammatory bowel disease management and design of an evaluation tool of nursing care
}

\author{
Antonio Torrejón ${ }^{1}$, Lorena Oltra², Paloma Hernández-Sampelayo ${ }^{3}$, Laura Marín ${ }^{4}$, Valle García-Sánchez ${ }^{5}$, \\ Francesc Casellas ${ }^{1}$, Noelia Alfaro ${ }^{6}$, Pablo Lázaro ${ }^{6}$ and María Isabel Vera ${ }^{7}$ \\ ${ }^{1}$ Unitat Atenció Crohn-Colitis. Hospital Universitari Vall d'Hebron. Centro de Investigación Biomédica de Red del Área \\ temática de Enfermedades Hepáticas y Digestivas (CIBERehd). Barcelona, Spain. ${ }^{2}$ Gastroenterology Service. Hospital de \\ Manises. Manises, Valencia. Spain. ${ }^{3}$ Gastroenterology Service. Hospital Universitario Gregorio Marañón. Madrid, Spain. \\ ${ }^{4}$ Gastroenterology Service. Hospital Universitario Germans Trias i Pujol. Badalona, Barcelona. Spain. ${ }^{5}$ Gastroenterology \\ Service. Hospital Universitario Reina Sofía. Córdoba, Spain. ${ }^{6}$ Técnicas Avanzadas de Investigación en Servicios de Salud \\ (TAISS). Madrid, Spain. ${ }^{7}$ Gastroenterology and Hepatology Department. Hospital Universitario Puerta de Hierro. \\ Majadahonda, Madrid. Spain
}

\section{ABSTRACT}

Background and aims: nursing management of inflammatory bowel disease (IBD) is highly relevant for patient care and outcomes. However, there is evidence of substantial variability in clinical practices. The objectives of this study were to develop standards of healthcare quality for nursing management of IBD and elaborate the evaluation tool "Nursing Care Quality in IBD Assessment" (NCQ-IBD) based on these standards.

Methods: a 178-item healthcare quality questionnaire was developed based on a systematic review of IBD nursing management literature. The questionnaire was used to perform two 2-round Delphi studies: Delphi A included 27 IBD healthcare professionals and Delphi B involved 12 patients. The NCQ-IBD was developed from the list of items resulting from both Delphi studies combined with the Scientific Committee's expert opinion.

Results: the final NCQ-IBD consists of 90 items, organized in 13 sections measuring the following aspects of nursing management of IBD: infrastructure, services, human resources, type of organization, nursing responsibilities, nurse-provided information to the patient, nurses training, annual audits of nursing activities, and nursing research in IBD. Using the NCQ-IBD to evaluate these components allows the rating of healthcare quality for nursing management of IBD into 4 categories: A (highest quality) through D (lowest quality).

Conclusion: the use of the NCQ-IBD tool to evaluate nursing management quality of IBD identifies areas in need of improvement and thus contribute to an enhancement of care quality and reduction in clinical practice variations.

Key words: Delphi technique. Inflammatory bowel disease. Nursing care. Standard of care. Total quality management.

Torrejón A, Oltra L, Hernández-Sampelayo P, Marín L, GarcíaSánchez V, Casellas F, Alfaro N, Lázaro P, Vera MI. Development of quality standards in inflammatory bowel disease management and design of an evaluation tool of nursing care. Rev Esp Enferm Dig 2013;105:262-271.

Received: $19-02-2013$

Accepted: 27-05-2013

Correspondence: Antonio Torrejón Herrera. Digestive Service. Hospital General Vall d'Hebron. Passeig Vall d'Hebron, 119-129. 08035 Barcelona, Spain e-mail: tonith@gmail.com

\section{INTRODUCTION}

Inflammatory Bowel Disease (IBD) is progressively becoming more prominent across industrialized nations' healthcare systems. For instance, Crohn's disease incidence reports in Europe fall between 2.5 and 7.5 cases per 100,000 population per year $(1,2)$. Based on recent years' increase in reported cases, current incidence rates are estimated at about 9 cases per 100,000 population per year (3-6).

Most cases of IBD -which include Crohn's disease and ulcerative colitis- present in young individuals during their formative or working-age years. This timing contributes significantly to IBD's major negative impact, both in the clinical sense and also in terms of the individual's quality of life (7). The extent of personal and socioeconomic consequences is reflected in the increasing consumption of healthcare resources, economic costs, and impact on work productivity (8). IBD treatment goals include: achieve remission and maximize length of remission, minimize medication side-effects, reduce symptoms, resolve any complications that may arise, and improve patients' quality of life. IBD management requires a multidisciplinary approach with interaction among doctors, nurses, surgeons, dieticians, and social workers, among others $(1,2)$.

Growing awareness of the key role of such multidisciplinary approach to IBD management has materialized in specific initiatives from some hospitals. Such initiatives have created general outpatient offices for nurse consultation and some have even developed Comprehensive Care Units for IBD (9-12). A previous study, specifically designed for Gastroenterology clinical centers, investigated different organizational models of nursing management of IBD in Spain, including responsibilities and resources available to IBD-specialized nursing. The findings of this 2009 survey showed significant heterogeneity regarding health care organizational nursing models for IBD and the impact on patients (13). Based on these findings, the research project described here was designed with two main objectives: First, to devel- 
op standards of quality to homogenize IBD nursing care; and second, based on said standards, to design a tool to evaluate the nursing care quality in IBD (NCQ-IBD).

\section{METHODS}

\section{Overall design}

Two Delphi studies were performed, one intended for healthcare professionals (Delphi A) and a second one aimed at patients (Delphi B)

Phase I - Delphi A (healthcare professionals)

The Delphi method is a consensus technique, consisting of a series (two or more) of consultations (rounds) by post or email to an expert panel, using questionnaires with questions that respondents answer by the first time (first round), without hints about what may respond the rest of the panel members. In a second round, there is a feedback in which each panelist is informed about the results of the previous round, and asked to respond again, in order to improve the degree of consensus among experts. This method ensures the anonymity for each respondent.

Our 2-round Delphi study for professionals took place between March and June of 2010 involving 27 experts from 9 of the 17 Autonomous Communities (regions) in Spain. Twelve experts were gastroenterologists, 12 nurses, and 3 surgeons (14). The questionnaire's 178 items stemmed from a systematic literature review on the effectiveness of the different existing organizational models of nursing management of IBD (15). One of the most relevant documents was the 2009 IBD standards Group document (16). The items selection was performed by the members of the project scientific committee (SC) formed by 3 gastroenterologists and 5 nurses.

The Delphi questionnaires included two kinds of items. The first type were statements reflecting recommendations (e.g., "Patient must receive a direct line telephone number") with possible answers on a scale of 9 points, where " 1 " stood for "not important at all" and "9" stood for "very important". The second type items were statements soliciting answers on a continuous scale (e.g., "Minimum amount of days per week that the IBD nursing unit should see patients"). The final questionnaire items were organized under 6 sections related to the IBD nursing care: Infrastructure (44 items); process (65 items); management and patient follow-up (38 items); specialized nurse training (23 items); nursing research (8 items); and suggestions section, where panel members were encouraged to make recommendations. Based on suggestions from panel members, the number of items for the second-round questionnaire was increased to 182 . In order to facilitate item re-evaluation during this round, the documentation received by each panel member included for each item response the mean, the standard deviation, and the panel member's own response to the item during the first round.

\section{Phase II - Delphi B (IBD patients)}

The patients' Delphi study was done between July and October 2010. The first-round questionnaires were sent to each member of the SC. This person, then, selected potential study participants randomly from all the patients based on the order at which they arrived to the nurse consultation office. The study participant inclusion criteria were: IBD patients 18 years of age or older, secondary school completed, at least five years since IBD diagnosis, and reporting at least two visits to the nursing care service in the previous year. Finally, 12 patients from six hospitals of five regions in Spain were selected as panel members. Second-round questionnaires were sent directly to each patient by electronic mail.

The SC designed the patients' questionnaire based on the healthcare professionals' questionnaire reducing the number of items, according to the ability of patients to respond, to 123. The items were organized in four sections: Infrastructure (35 items); process (60 items); management and patient follow-up (22 items); and specialized nurse training (6 items).

\section{Phase III - Selection of standard of quality items}

Once data from professionals and patients Delphi studies were analyzed, all quality of care standard items were combined into a single list resulting in 193 items. This standards-of-quality item list became the working document used by the SC to rate each item into one of three categories: high, medium, or low importance for measuring the quality of care. The first step in the classification process was to rate items based on the scores assigned by panel members while weighing the relative importance of health professionals' contributions vs. patients' contributions. Before the rating and weighing process started, however, all 193 items were organized into 16 sections to group items by response scale (1-9 scale vs. continuous) as well as by topic (infrastructure, process, etc.).

- Categories: Cut-off points. In a first meeting, the SC established classification criteria to use as reference points in the rating of the standards of quality in order to facilitate scoring each of the 16 sections. The SC agreed on 3 cut-off points for each of the items on scales from 1 to 9 for assigning the importance for measuring the quality of care: high, medium, or low importance. Items scored the highest by panel members were considered highly important, fundamental or basic, whereas the ones receiving the lowest scores were labeled as low important for quality aspects. The items ranked below "low importance" were eliminated in the NCQ-IBD. For continuous scale items two complementary percentiles (i.e., both percentiles must add up to 1) were used to define three quality categories for each item: Excellent, medium, and basic. For instance, for item 11 "Available on-demand nursing 
appointments for IBD days per week" the 0.16 and 0.84 percentiles were used resulting in "Excellent" above 4.4, "Basic" below 2.5, and "Medium" between 2.5 and 4.4 days per week.

- Data weighting. The SC used weights to assign more or less relevance to the answers from each panel (professionals or patients) according to the item's topic. $\mathrm{SC}$ experts assigned weights between 0 and 1 to healthcare professionals' scores (healthcare professional weight), and a complementary weight (1healthcare professionals weight) to patients' score. Thus, for instance, a weight of 1 meant that only healthcare professionals' opinions were deemed relevant, a weight of 0 meant that only patients' opinions were deemed relevant, and a weight of 0.50 for each score meant that both opinions were deemed equally relevant. The sum of the assigned weights to healthcare professionals and to patients had to add up to 1 .

In order to avoid SC members' opinions biasing each other's scoring, each member was given a folder with a copy of the questionnaire so that they could score each section privately. Each SC member entered their score in the corresponding boxes. From these values the mean was then calculated and used as reference point for the rating and weighing of the standards of quality.

During a second meeting, items considered dispensable (with a final score below the low importance cut-off point) by SC experts were eliminated to make the instrument more efficient. Items specific to nursing management of IBD were kept, together with the most objective items. Finally, this streamlined version was used to build the NCQ-IBD tool for the evaluation of nursing care quality in IBD.

\section{RESULTS}

Finally, 90 items (quality of care standards) were elaborated; table I shows the final cut-off points used for rating the item importance (items on scales from 1 to 9), the item excellence category (continuous scale items), the weighing values, as well as the number of final items for each section. The NCQ-IBD kept 90 of the initial 193 items. The first column displays the heading of the section of the list of items resulting from merging the healthcare professional and patient Delphi questionnaires. The second column shows the corresponding number of items. Items included in the questionnaire after eliminating the dispensable ones are specified in the third column. The three following columns present the SC-assigned cut-off points used for rating the items importance and excellence categories. Weighing values are specified next. Items included in just one of the Delphi questionnaires (answered only by either healthcare professionals or by patients) do not require weighing values. For building the NCQ-IBD tool (Appendix 1), each quality standard was operationalized into a specific, quantifiable question. The final NCQ-IBD tool is comprised of 90 items organized in 13 sections. For prac- tical use, the NCQ-IBD is supplemented by an instruction manual specifying who must complete the instrument and how to respond to the questions. It also includes a glossary of terms to avoid ambiguity in the interpretation of items.

Once the NCQ-IBD was designed, the SC met to agree upon a rating of nursing healthcare quality in IBD to facilitate the operationalization of the quality standards instrument using the items values for assigning a quality of nursing care level for a specific IBD service. The SC accomplished the task of assigning final ratings to the different models of nursing healthcare based on item grouping. First, the SC defined quality standards at three levels of quality of care. Items on scales from 1 to 9 were assigned one of three labels: Level IS-Basic (high importance), Level IIS-Medium (medium importance), or Level IIIS-Excellent (low importance). Continuous items were assigned similar labels: Level IC-Basic, Level IIC-Medium, or Level IIICExcellent (column 3, Appendix 1). This classification reflects the importance the panel members assigned to the 1-9 scale indicators and the minimum criteria assigned to continuous indicators. Out of the 90 items included in the list of care standards were $671-9$ scale and 23 were continuous. Out of the 67 1-9 scale items panel members ranked 20 as level IS or Basic, 33 as level IIS or Medium, and 14 as level IIIS or Excellent. In regards to the care standard, criteria for level IS-IC represent the very basic, i.e., the minimum level expected for any rating in quality healthcare. Criteria for level IIS-IIC items correspond to medium healthcare quality; and Level IIIS-IIIC items denote excellent quality care.

As it may be that a particular service was well evaluated fulfilling correctly many low relevant items, the SC decided to set items that should necessarily be met for each level of quality care. For this reason, it was considered that the quality level of nursing care in IBD depends on two conditions: a) Meeting specific required items at each of the levels examined (Table II); and b) totaling to a minimum number of items for reaching a determinate quality level (Table III). Accordingly, once the items were classified by levels four categories for "Nursing care quality in IBD" were created labeled A, B, C, and D. Category A corresponds to the highest quality nursing care model and category D corresponds to the lowest quality model. Criteria for each of these four categories are described in table III. Thus, for those cases where either one of the conditions are not met, the quality of nursing care model will be placed in the category according to the highest common rating reached in both set of criteria illustrated in tables II and III.

\section{DISCUSSION}

Research describing nursing best practices in IBD is scarce. Thus, this study explores quality standards that allow identify responsibilities that nursing should foster as well as the resources they should have available to potentially improve quality and patients outcomes. As part of this 
Table I. Ratings and weights of the different measurement sections to evaluate nursing care quality in IBD

\begin{tabular}{|c|c|c|c|c|c|c|c|c|c|c|}
\hline \multirow{2}{*}{ Indicators } & \multirow{2}{*}{$\begin{array}{l}\text { \# of items by } \\
\text { Section (193) }\end{array}$} & \multirow{2}{*}{$\begin{array}{l}\text { \# of final } \\
\text { items (90) }\end{array}$} & \multicolumn{6}{|c|}{ Cut-off points used for rating the item importance } & \multicolumn{2}{|l|}{ Weights } \\
\hline & & & \multicolumn{3}{|c|}{ Scale 1 to 9 (S) } & \multicolumn{3}{|c|}{ Continuous (C) } & $\begin{array}{l}\text { Healthcare } \\
\text { professionals }\end{array}$ & Patients \\
\hline
\end{tabular}

Infrastructure

\begin{tabular}{|c|c|c|c|c|c|c|c|c|c|c|}
\hline Environment of IBD care ${ }^{1}$ & 3 & 0 & NA & & & $<0.25$ & $0.25-0.75$ & $>0.75$ & NA & \\
\hline Minimum IBD-related services ${ }^{1}$ & 4 & 3 & NA & & & $<0.16$ & $0.16-0.84$ & $>0.84$ & 0.51 & 0.49 \\
\hline Space identification and description² & 14 & 3 & 7.00 & 7.50 & 8.19 & NA & & & 0.49 & 0.51 \\
\hline Nurse Consultation Office resources ${ }^{2}$ & 11 & 6 & 7.86 & 8.33 & 8.71 & NA & & & 0.51 & 0.49 \\
\hline Human resources: patients ${ }^{2}$ & 8 & 0 & 7.50 & 8.00 & 8.50 & NA & & & Only professionals & Only patients \\
\hline Specialists available for referral ${ }^{2}$ & 7 & 4 & 7.13 & 7.63 & 8.19 & NA & & & 0.54 & 0.46 \\
\hline
\end{tabular}

Process

\begin{tabular}{llllllllll}
\hline Nursing staff responsibilities $^{2}$ & 38 & 27 & 7.13 & 7.69 & 8.13 & NA & & 0.59 & 0.41 \\
Organization of IBD management $^{1}$ & 17 & 8 & NA & & & $<0.23$ & $0.23-0.77$ & $>0.77$ & 0.54 \\
Organization to contact IBD patients $^{2}$ & 14 & 4 & 7.50 & 8.00 & 8.50 & NA & & 0.46 & 0.53 \\
\hline
\end{tabular}

Management and patient follow-up

\begin{tabular}{|c|c|c|c|c|c|c|c|c|}
\hline IBD management quality indicators² & 25 & 6 & 6.29 & 7.07 & 7.93 & NA & 0.64 & 0.36 \\
\hline Information to patient provided by nurses ${ }^{2}$ & 11 & 6 & 7.07 & 7.57 & 8.07 & NA & 0.39 & 0.61 \\
\hline
\end{tabular}

Training

\begin{tabular}{llllllllll}
\hline Training plan' $^{2}$ & 20 & 10 & 7.00 & 7.50 & 8.00 & NA & & 0.51 & 0.49 \\
Training time $^{1}$ & 5 & 3 & NA & & & $<0.23$ & $0.23-0.77$ & $>0.77$ & Only professionals
\end{tabular}

Research

\begin{tabular}{|c|c|c|c|c|c|c|c|c|}
\hline Research responsibility² & 3 & 1 & 7.64 & 8.04 & 8.37 & NA & & Only professionals \\
\hline Research performed $^{1}$ & 5 & 3 & NA & & & $<0.41$ & $0.41-0.59>0.59$ & Only professionals \\
\hline
\end{tabular}

IBD: Inflammatory bowel disease; 1Continuous; 2Measure evaluation scale from 1 to 9 ( 1 = not important at all, 9 = very important); NA: Not applicable.

investigation, two Delphi studies were carried out targeting both healthcare professionals and patients. Based on the information provided by these studies, a list was compiled which included care standards regarding resources, processes, training and research, among others. The NCQ-IBD was designed from such list of quality standards. This tool allows an assessment rating the quality of care provided by nurses working at IBD services. The NCQ-IBD categorizes nursing quality of care into four categories from A (highest) to D (lowest).

Results of an earlier survey identified both the responsibilities fulfilled by nurses as well as the resources available for the nursing management of IBD in Spain. Among the main findings, significant effects different nursing organizational structures have on IBD patients' outcomes were reported (13). The paucity of similar instruments calls for the development of tools such as the NCQ-IBD to facilitate assessing current nursing management approaches to IBD care. The application of this tool will assist generate strategies for the improvement of IBD management.

These findings as well as the instrument should be assessed within the context of potential limitations not uncommon in Delphi studies and instrument design. For instance, panel members may not fully represent the populations of interest since they were not selected by randomized sampling. They represent a convenience sample, in which some selection bias may have occurred. To maximize representation -within the scope of the study-data from both healthcare professionals and patients across the country and care levels were collected. Since the final panel was composed of 27 healthcare professionals (12 gastroenterologists, 12 nurses, and 3 surgeons) and 12 pa- 
Table II. Criteria needed for the rating of nursing care quality in IBD

Nursing care quality standards in inflammatory bowel disease

Specific items needed for the rating of nursing care quality in IBD

\begin{tabular}{|c|c|c|c|}
\hline$D$ & $C$ & B & $A$ \\
\hline & \multirow[t]{2}{*}{$x$} & $x$ & $x$ \\
\hline & & $x$ & $x$ \\
\hline & $x$ & $x$ & $x$ \\
\hline & $<2$ & $4-2$ & $>4$ \\
\hline & $>0 \leq 0.5$ & $\geq 0.5 \leq 1$ & $\geq 1$ \\
\hline & \multirow{4}{*}{$>12$} & $6-12$ & $<6$ \\
\hline & & $x$ & $x$ \\
\hline & & & $x$ \\
\hline & & & $x$ \\
\hline & \multirow[t]{3}{*}{$x$} & $x$ & $x$ \\
\hline & & $x$ & $x$ \\
\hline & & & $x$ \\
\hline & \multirow[t]{4}{*}{$x$} & $x$ & $x$ \\
\hline & & $x$ & $x$ \\
\hline & & $20-40$ & $>40$ \\
\hline & & $x$ & $x$ \\
\hline & 5 & 13 & 16 \\
\hline
\end{tabular}

IBD: Inflammatory bowel disease; A: Highest quality nursing care model; D: Worst quality nursing care model (fails to meet minimum requirements for category C).

Table III. Rating of nursing care quality in IBD

\begin{tabular}{|c|c|c|c|c|c|c|c|}
\hline \multirow[b]{3}{*}{$\begin{array}{l}\text { HEALTHCARE } \\
\text { quality level }\end{array}$} & \multicolumn{6}{|c|}{ Number of items needed for each level } & \multirow[b]{3}{*}{$\begin{array}{l}\text { Specific } \\
\text { required itemse }\end{array}$} \\
\hline & \multicolumn{3}{|c|}{ Scale 1 to $9(S)$} & \multicolumn{3}{|c|}{ Continuous $(C)^{d}$} & \\
\hline & Items I-Sa & Items II-S & Items III-Sc & Items I-C & Items II-C & Items III-C & \\
\hline C & 10 & & & & & & $C(5)$ \\
\hline D & Lower than $\mathrm{C}$ & & & & & & D \\
\hline
\end{tabular}

IBD: Inflammatory bowel disease; A: Highest quality nursing care model; D: Lowest quality nursing care model (fails to meet minimum requirements for category C). ${ }^{2} 20$ available items; ${ }^{\text {b3 } 3}$ available items; ' 14 available items; ${ }^{\circ} 23$ available items. 'Rating of nursing care quality must satisfy the specific required item of the corresponding category (see table II). ${ }^{\mathrm{f}}$ At least 12 items from level II-C and/or III-C must be satisfied.

tients from nine Spanish regions, the risk of a biased expert panel and, thus, skewed scores leading to invalid results, is extremely low.

Additionally, the set of indicators included may not be comprehensive. However, this is highly unlikely since the instrument includes the most relevant items for its intent due to the methods followed: a systematic search for standard indicators in relevant literature; a preliminary compilation of identified standard indicators describing different nursing management models of IBD; and the SC's revision of the list of identified standard indicators. Further, another potential threat to the creation of standard indicators is the influence experts may exert on each other's opinions. In this study this source of bias was eliminated by using the Delphi method. By this method, experts only interact during the second round of the study and responses are kept confidential throughout. The development of similar instruments in previous Delphi studies establishes the validity of this methodology (17-21). Finally, the instrument's main limitation resides in its rating system based on the SC's expert assessment since the rating system is pending of being applied in the real world. 
Among the project's strengths is that the best suited methodology for the design of a measure of quality of care such as NCQ-IBD was followed starting with a rigorous systematic review of literature about the effectiveness of the different nursing management models and behaviors in IBD (15). Based on the analysis of this empirical evidence, two separate Delphi questionnaires were designed for both healthcare professionals with extensive IBD experience and patients with at least five years from diagnosis to evaluate standards of structure, process, research and training. Additionally, once validated, the NCQ-IBD will fill in the gap in the literature of validated indicators of IBD care quality uncovered by the systematic review. Motivated by the patent need for measure development in this specialized field, through this study the most relevant standard indicators of quality were selected, operationalized, and combined into a user-friendly instrument that assesses the nursing care quality in IBD.

The standards developed in this study have contributed to the identification of a set of requirements -such as resources, processes, and training and research-needed by nursing to provide satisfactory levels of IBD care. The
NCQ-IBD could be an effective tool to evaluate the healthcare provided specifically by nursing. Based on its ratings, minimum acceptable quality standards in healthcare, research, and training can be identified and outlined. Therefore, the NCQ-IBD provides a powerful tool to reduce large amounts of information into useful specific quality standards. These standards can then be applied by healthcare providers, patient associations, and the Healthcare Administration to homogenize IBD management protocols, reduce clinical practice variations, and improve nursing care in IBD. Ultimately, these improvements will result in better outcomes for IBD patients.

\section{ACKNOWLEDGEMENTS}

The Delphi study and the instrument development research were supported by an unrestricted grant from Abbott Laboratories. Research and reports were produced independently by the authors, with full editorial control resting with the authors. Abbott Laboratories did not participate in any part of the study.

Appendix. Nursing Care Quality in IBD Assessment (NCQ-IBD)

\section{N. ${ }^{\circ}$ of \\ items \\ $90 \quad$ NURSING CARE QUALITY IN INFLAMMATORY BOWEL DISEASE ASSESSMENT (NCQ-IBD)}

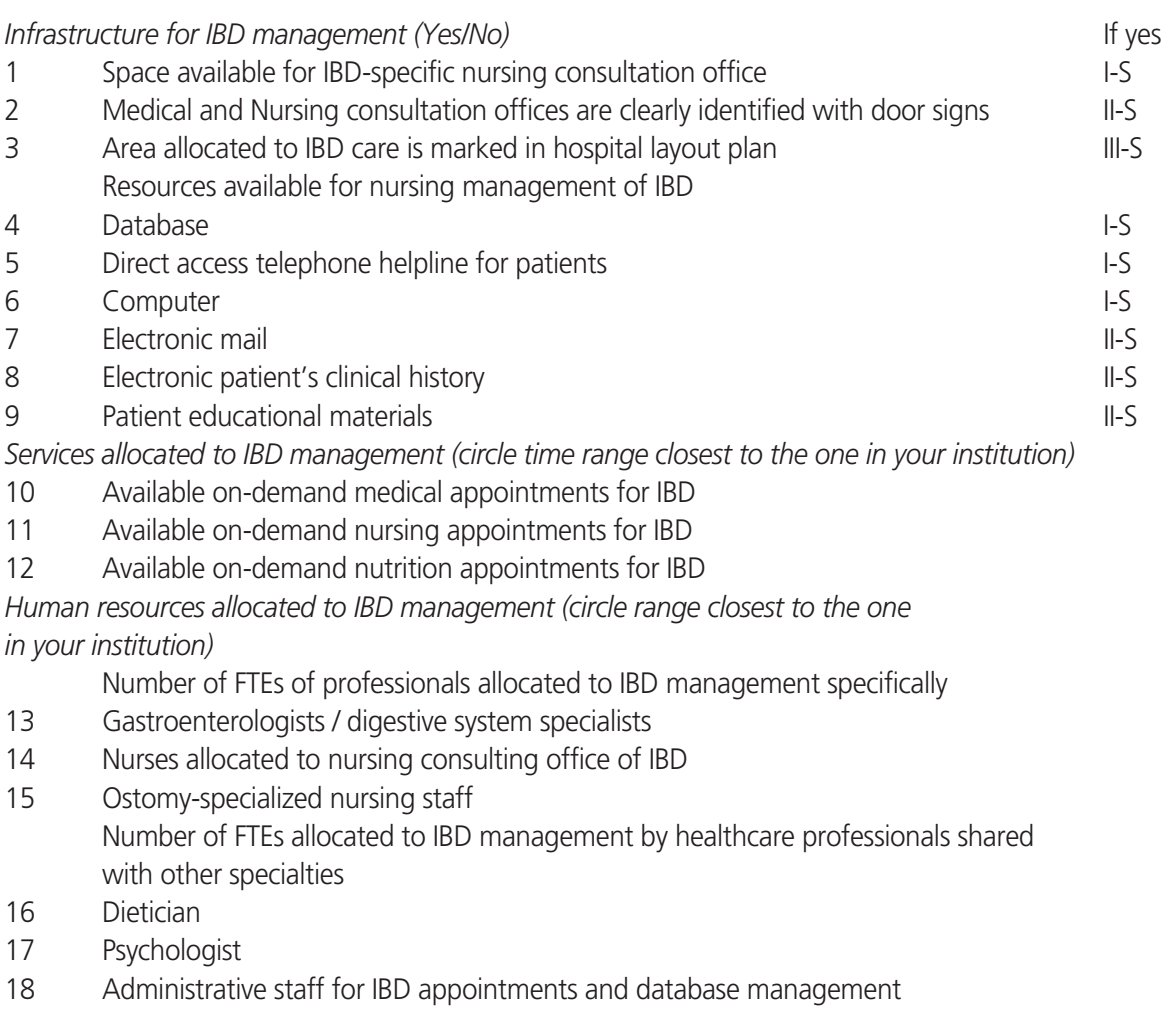

If yes

$-S$

$S$

$-S$

$1-S$

Item Rating

\begin{tabular}{llllll}
\hline I-S & H-S & II-S & I-C & I-C & III-C
\end{tabular}

Number of days per week

$<2.5 \quad 2.5-4.5 \quad>4.5$

$<2.5 \quad 2.5-4.4 \quad>4.4$

$\begin{array}{lll}<1 & 1\end{array}$

Number of FTES

$\begin{array}{lll}<1 & 1-2 & >2 \\ <1 & 1 & >1 \\ <1 & 1 & >1 \\ & & \\ & & \\ <0.5 & 0.5-1 & >1 \\ <0.5 & 0.5-1 & >1 \\ <0.5 & 0.5-1 & >1\end{array}$


Appendix 1 (Cont.) Nursing Care Quality in IBD Assessment (NCQ-IBD)

\begin{tabular}{ll}
\hline $\begin{array}{l}N^{\circ} \text { of } \\
\text { items }\end{array}$ & \\
\hline $90 \quad \begin{array}{l}\text { NURSING CARE QUALITY IN INFLAMMATORY BOWEL DISEASE } \\
\text { ASSESSMENT (NCQ-IBD) }\end{array}$ \\
\cline { 1 - 2 }
\end{tabular}

Specialists available for referrals (Yes/No)

Specialists available for referrals with IBD experience such as:

19 Gastrointestinal surgeon

20 Rheumatologist

21 Obstetrician/gynecologist

22 Dermatologist

Organization in IBD management (circle time range closest to the one in your institution)

23 Hours per day allocated to outpatient consultation with a nurse in IBD

24 Minutes allocated to first outpatient consultation with a nurse

25 Minutes allocated to following outpatient consultations with a nurse

26 Minutes allocated to first outpatient consultation with a nurse post-ostomy

27 Minutes per day of outpatient consultation with a nurse to attend walk-in patients

28 Minimum number of times per year that nursing staff should assess IBD patients Maximum IBD patient waiting time:

29 Wait time (in working days) for first regular appointment with nursing

30 Wait time (in minutes) in nursing office's waiting room

Organization to contact IBD patient (Yes/No)

Information given to the patient:

31 Direct access telephone helpline for patients

$32 \quad$ Name of nurse contact

33 Patient must be asked for contact telephone number

$34 \quad$ IBD nurses prioritize needs originating from patients calling nursing

Nursing responsibilities in IBD management (Yes/No)

35 Educate patients in medication self-administration

36 Perform regular follow-up of patients according to each type of treatment

37 Monitor follow-up and adherence to biologic drug treatments

Monitor follow-up and adherence to non-biologic drug treatments

Supervise blood work between visits for those drugs requiring monitoring

Review medication during consultation: drug information, secondary effects

(in short- and long-term)

41 Modify treatment protocol per agreement between physician and nursing staff

59 Supervise the observation of diagnostic tests completion
Item Rating 
Appendix 1 (Cont.) Nursing Care Quality in IBD Assessment (NCQ-IBD)

\begin{tabular}{ll}
$\begin{array}{l}\text { N. }{ }^{\circ} \text { of } \\
\text { items }\end{array}$ & \\
\hline $90 \quad$ NURSING CARE QUALITY IN INFLAMMATORY BOWEL DISEASE \\
& ASSESSMENT (NCQ-IBD)
\end{tabular}

60 Take patient clinical history and perform physical exam to assess IBD symptoms

61 Administer questionnaires, tests, and indices to learn the patients' biopsychosocial characteristics

Information to patient provided by nurses (Yes/No) Nursing:

62 Nursing must report resources available for IBD management (e.g., consultation office hours, operation)

63 Informs patients about diagnostic tests performed

64 Provides verbal plain-language information regarding the disease status, its progress, and physical exams

65 Provides written plain-language information regarding the disease status, its progress, and physical exams

66 Provides didactic audio or video materials or webpage address covering diagnosis, treatment and self-care information

67 Provides contact information for relevant patient groups or associations.

Quality indicators in IBD management (Yes/No)

68 Nurses apply CPG or protocols

Assess aspects of the patient's biopsychosocial characteristics such as:

69 Classification of IBD

$70 \quad$ IBD activity indices

71 Treatment Compliance Tests

72 Quality of life Questionnaires

73 Depression and anxiety scales

IBD training for nurses (Yes/No)

$74 \quad$ Training plan in place for IBD-specialized nursing staff

Nursing staff are trained specifically in:

$75 \quad$ IBD clinical aspects

76 Skills in communication, patient support, and stress management

77 Ostomy care

$78 \quad$ Nutrition and diets

Nursing staff receive training in:

79 IBD classification

$80 \quad$ IBD activity indices

81 Treatment compliance tests

82 Quality of life questionnaires

83 Depression and anxiety scales

Time allocated to IBD training (circle time range closest to the one in your institution) Hours a nurse has allocated to training in previous two years:

$84 \quad$ IBD

85 Ostomies

86 Clinical sessions nursing participates in per month

Annual reviews of IBD nursing activity and outcomes (Yes/No)

$87 \quad$ Nursing reviews its activities and outcomes annually

Nursing research in IBD (Circle range closest to the one in your institution)

In the last 5 years nursing has participated in:

88 Research projects

89 Conference presentations

90 Journal publications
III-S

III-S

If yes

I-S

$\|-S$

II-S

II-S

III-S

III-S

If yes

II-S

$\|-S$

II-S

II-S

III-S

III-S

If yes

I-S

I-S

I-S

I-S

$\|-S$

$\|-S$

II-S

III-S

III-S

Number of:

$\begin{array}{lll}<40 & 40-60 & >60 \\ <20 & 20-35 & >35 \\ <1 & 1-2 & >2\end{array}$

If yes

II-S

Number:

$\begin{array}{lll}<3 & 3-5 & >5 \\ <4 & 4-5 & >5 \\ <2 & 2-3 & >3\end{array}$




\section{REFERENCES}

1. Shivananda S, Lennard-Jones J, Logan R, Fear N, Price A, Carpenter L, et al. Incidence of inflammatory bowel disease across Europe: is there a difference between north and south? Results of the European Collaborative Study on Inflammatory Bowel Disease (CD-IBD). Gut 1996;39:690-7.

2. Saro Gismera C, Lacort Fernández M, Argüelles Fernández G, Antón Magarzo J, García López R, Navascues CA, et al. Incidencia y prevalencia de la enfermedad inflamatoria intestinal en Gijón, Asturias, España. Gastroenterol Hepatol 2000;23:322-7.

3. Nerich V, Monnet E, Etienne A, Louafi S, Ramée C, Rican S, et al. Geographical variations of inflammatory bowel disease in France: A study based on national health insurance data. Inflamm Bowel Dis 2006;12:218-26.

4. Sinci BM, Vuceli B, Persi M, Brnci N, Erzen DJ, Radakovi B, et al. Incidence of inflammatory bowel disease in Primorsko-goranska County, Croatia, 2000-2004: A prospective population-based study. Scand J Gastroenterol 2006;41:437-44.

5. Vind I, Riis L, Jess T, Knudsen E, Pedersen N, Elkjaer M, et al. Increasing incidences of inflammatory bowel disease and decreasing surgery rates in Copenhagen City and County, 2003-2005: a population-based study from the Danish Crohn colitis database. Am J Gastroenterol 2006; 101:1274-82.

6. Saro C, de la Coba C, Lacort M, et al. Changes in Incidence of Inflammatory Bowel Disease during the Last 15 years: A hospitalization al prospective population-based study from Gijón (Spain) (1992-2006). Gut 2007;39(Supl. 1):A129.

7. Casellas F, Arenas JI, Baudet JS, Fábregas S, García N, Gelabert J, et al. Impairment of health-related quality of life in patients with inflammatory bowel disease: A Spanish multicenter study. Inflamm Bowel Dis 2005;11:488-96.

8. Vergara M, Montserrat A, Casellas F, Villoria A, Suarez D, Maudsley $\mathrm{M}$, et al. A new validation of the Spanish Work Productivity and Activity Impairment Questionnaire-Crohn's disease version. Value Health 2011;14:859-61.

9. Fontanet G, Casellas F, Malagelada JR. La Unidad de Atención CrohnColitis: 3 años de actividad. Gac Sanit 2004;18:483-5.

10. Consulta de Enfermería pionera en Galicia. Enfermería avanza, 6 de julio de 2007 ( sede web). Disponible en: http://enfeps.blogspot.com/ 2007/07/consulta-de-enfermera-pionera-en.html (Acceso 29 de octubre de 2008).

11. Torrejón Herrera A, Masachs Peracaula M, Borruel Sainz N, Castells Carner I, Castillejo Badía N, Malagelada Benaprés JR, et al. Aplicación de un modelo de asistencia continuada en la enfermedad inflamatoria intestinal: la Unidad de Atención Crohn-Colitis. Gastroenterología y Hepatología 2009;32:77-82.

12. Mowat C, Cole A, Windsor A, Ahmad T, Arnott I, Driscoll R, et al. Guidelines for the management of inflammatory bowel disease in adults. Gut 2011;60:571-607.

13. Marín L, Torrejón A, Oltra L, Seoane M, Hernández-Sampelayo P, Vera MI, et al. Nursing resources and responsibilities according to hospital organizational model for management of inflammatory bowel disease in Spain. J Crohns Colitis 2011;5:211-7.

14. Fitch K, Berstein SJ, Aguilar MD, et al. The RAND/UCLA Appropriateness Method User's Manual. Santa Monica: RAND; 2001. Report No. MR-1269-DG-XI/RE.

15. Hernández-Sampelayo P, Seoane M, Oltra L, Marín L, Torrejón A, Vera MI, et al. Contribution of nurses to the quality of care in management of inflammatory bowel disease: A synthesis of the evidence. J Crohns Colitis 2010;4:611-22.

16. IBD Standards Group. "Quality care: Service standards for the healthcare of people who have Inflammatory Bowel Disease (IBD)". The IBD Standards Group, 2009.

17. Shekelle PG, Chassin M, Park RE. Assessing the predictive validity of the RAND/UCLA appropriateness method criteria for performing carotid endarterectomy. Int J Technol Assess Health Care 1998;14:70727.

18. Shekelle PG, Kahan JP, Bernstein SJ, Leape LL, Kamberg CJ, Park RE. The reproductibility of a method to identify the overuse and underuse of medical procedures. N Engl J Med 1998;338:1888-95.

19. McDonnell J, Meijler A, Kahan JP, Bernstein SJ, Rigter H. Panelist consistency in the assessment of medical appropriateness. Health Policy 1996;37:139-52.

20. Kravitz RL, Laouri M, Kahan JP, Guzy P, Sherman T, Hilborne L, et al. Validity of criteria used for detecting underuse of coronary revascularization. JAMA 1995;274:632-8.

21. Shekelle PG. Are appropriateness criteria ready for use in clinical practice? N Engl J Med 2001;344:677-8. 